\title{
Contaminación por Escherichia coli Shigatoxigénica en Puestos de Expendio de Carne de Pollo en un Distrito de Lima
}

\author{
Shigatoxigenic Escherichia coli Contamination in Chicken Meat Stalls \\ in a District of Lima, Peru \\ Juan Raúl Lucas L. ${ }^{1}$, Siever Morales Cauti ${ }^{2,5}$, Erika Paloma Salazar Jiménez ${ }^{4}$, \\ Carlos Eslava Campos ${ }^{4}$, Débora E. Alvarado ${ }^{3}$
}

\section{Resumen}

El objetivo del presente trabajo fue determinar si los puestos de venta de carne de pollo son una fuente de contaminación con Escherichia coli shigatoxigénica (STEC) en mercados de abastos. Se tomaron hisopados de la superficie de manos, tablas de picar y mesas de 50 puestos de expendio de carne de pollo en el distrito de San Juan de Miraflores, Lima, Perú ( $\mathrm{n}=150$ muestras). Se realizó aislamiento microbiológico estándar e identificación molecular de los genes stx 1 , stx2 y eaeA mediante PCR. El 42\% (63/150) y 25.3\% (38/ $150)$ de las muestras fueron positivas a $E$. coli y STEC, respectivamente. El $84 \%(42 / 50)$ y $66 \%$ (33/50) de los puestos de venta poseían al menos una de las superficies contaminadas con E. coli y STEC, respectivamente. El 68.3\% (43/63) de las cepas de E. coli aisladas fueron patógenas por presentar al menos un gen evaluado. De estas, 38 cepas fueron STEC y presentaron los genes stx $1(19.0 \%, 12 / 63)$, stx $2(14.3 \%, 9 / 63)$ y las asociaciones: $s t x 1$ y $s t x 2(12.7 \%, 8 / 63), s t x 1, s t x 2$ y eaeA $(6.3 \%, 4 / 63), s t x 2$ y eaeA $(4.8 \%, 3 / 63)$, y stx 1 y eaeA $(3.2 \%, 2 / 63)$. Se observaron prácticas de higiene deficientes en el puesto de venta y durante el expendio. Se confirma que los puestos de venta de carne de pollo del mercado limeño son fuente potencial de contaminación de STEC.

Palabras clave: Escherichia coli, mesa de expendio, tablas de picar, patógeno, prácticas de higiene, STEC

\footnotetext{
${ }^{1}$ Centro de Investigaciones IVITA, Estación El Mantaro, Universidad Nacional Mayor de San Marcos, Huancayo, Perú

${ }^{2}$ Laboratorio de Microbiología y Parasitología Veterinaria, Facultad de Medicina Veterinaria, Universidad Nacional Mayor de San Marcos, Lima, Perú

${ }^{3}$ Laboratorio Microbiología Molecular y Biotecnología, Facultad de Ciencias Biológicas, Universidad Nacional Mayor de San Marcos, Lima, Perú

${ }^{4}$ Laboratorio de Bacteriología Intestinal, Hospital Infantil Federico Gómez, D.F. México, México

${ }^{5}$ E-mail: sieverm@hotmail.com
} 
The aim of this study was to determine if chicken meat stalls are sources of contamination with shigatoxigenic Escherichia coli (STEC) in Lima, Peru. Swabs from the surfaces of hands, cutting boards and sale tables of 50 chicken meat stalls in a large market in the district of San Juan de Miraflores, Lima, Peru were taken ( $\mathrm{n}=150$ samples). Standard microbiological isolation and molecular identification of stx 1 , st $x 2$ and eaeA genes by PCR was performed. Results showed that $42 \%$ (63/150) and 25.3\% (38/150) of samples were positive for $E$. coli and STEC respectively. Besides, 84\% (42/50) and 66\% $(33 / 50)$ of chicken meat stalls had at least one contaminated surface with $E$. coli and STEC respectively. Also, $68.3 \%$ (43/63) of strains of $E$. coli isolated were pathogenic for presenting at least one of the evaluated genes. There were 38 STEC strains and presented stx $1(19.0 \%, 12 / 63)$, stx $2(14.3 \%, 9 / 63)$ and the associations stx 1 and stx $2(12.7 \%, 8 / 63)$, $s t x 1, s t x 2$ and eaeA $(6.3 \%, 4 / 63)$, stx 2 and eae $\mathrm{A}(4.8 \%, 3 / 63)$, and $s t x 1$ y eae $\mathrm{A}(3.2 \%, 2 / 63)$. Poor hygiene practices were observed in the chicken meat retail stores. It is confirmed that chicken meat stalls in the Lima market were potential sources of contamination of STEC.

Key words: Escherichia coli, sale table, cutting board, pathogen, hygiene practices, STEC

\section{INTRODUCCIÓN}

Escherichia coli es una bacteria que forma parte de la microflora intestinal de los humanos y animales. Sin embargo, algunas cepas de $E$. coli han adquirido factores de virulencia que las hacen patógenas, denominándose cepas diarrogénicas o patógenicas, las cuales se clasifican en seis patotipos o subgrupos (Nataro y Kaper, 1998). E. coli shigatoxigénica (STEC) es el patotipo más común causante de enfermedades trasmitidas por alimentos (ETA) que ha emergido en los últimos años (Gyles, 2007).

Las STEC poseen las shigatoxinas (Stxs), Stx1 y Stx2, que son factores de virulencia de naturaleza proteica, codificados en los genes $s t x 1$ y stx 2 . Stxs producen colitis hemorrágica, pero también conllevan una acción sistémica que puede causar síndrome urémico hemolítico (SUH), una severa secuela que se caracteriza por falla renal, anemia hemolítica y trombocitopenia. La intimina (del gen eaeA) es otro factor de virulencia importante que puede estar presente en STEC y mediante el cual se adhiere al enterocito (Gyles, 2007).

Los niños menores de 5 años y los ancianos constituyen el grupo más vulnerable, con una mayor incidencia de infecciones sintomáticas por STEC y alto riesgo de evolución a SUH (Thorpe, 2004). En el Perú, STEC se ha asociado al 9.2\% de los cuadros de diarrea sanguinolenta en niños de menos de 5 años (Llanos et al., 2012).

El principal reservorio de STEC son los rumiantes domésticos. Aparentemente el pollo y su carne no son reservorios importantes de STEC, encontrándose prevalencias de menos de 1\% (Read et al., 1990; Piérard et al., 1997; Xia et al., 2010). Sin embargo, se ha reportado que el manejo en el puesto de venta incrementa la frecuencia de STEC en la carne debido a la contaminación cruzada durante su manipulación (Samadpour et al., 1994; Chinen et al., 2009; Etcheverria et al., 2010; Alonso et al., 2012). 
Por lo expuesto, es importante confirmar si las actividades de expendio son una fuente de contaminación con STEC en uno de los más grandes mercados de abastos de Lima, Perú, especialmente si se considera que la carne de pollo es la de mayor consumo en el Perú y es también vehículo de ETA (Lucas et al., 2013; Zambrano et al., 2013).

\section{Materiales y Métodos}

\section{Lugar de Estudio y Muestreo}

Se evaluaron los puestos de venta de carne de pollo del Mercado Cooperativo de Ciudad de Dios, del distrito de San Juan de Miraflores, Lima, Perú. Se muestreó la totalidad de puestos de venta de carne de pollo $(n=50)$ en marzo del 2013. El estudio se hizo en coordinación con la Gerencia de Salud Pública de la Municipalidad Distrital y los dirigentes del mercado de abasto.

Se tomaron tres muestras por puesto de venta con el uso de hisopos estériles. Estas fueron muestras de manos (hisopado de ambas manos), tablas de picar (hisopado de la superficie que tiene contacto con la carne) y mesa de expendio (hisopado de la mesa alrededor de la tabla), resultando en 150 muestras en total. Los hisopos se llevaron en medio de transporte Stuart y en caja térmica a $4{ }^{\circ} \mathrm{C}$ hasta el laboratorio de Microbiología de la Facultad de Medicina Veterinaria y Zootecnia de la Universidad Científica del Sur para la identificación microbiológica de $E$. coli y detección de los factores de virulencia determinantes de STEC.

Durante la toma de muestras se observaron las posibles faltas en las medidas de higiene presentes en cada uno de los puestos.

\section{Identificación de Escherichia coli}

La identificación de $E$. coli se realizó de forma convencional (MINSA, 2005), la misma que incluyó un enriquecimiento en caldo tripticasa de soya $\left(24 \mathrm{~h}, 37^{\circ} \mathrm{C}\right)$ y aisla- miento en agar McConkey $\left(24 \mathrm{~h}, 37^{\circ} \mathrm{C}\right)$ de colonias lactosa positivas. Estas cepas fueron evaluadas mediante pruebas bioquímicas de rojo de metilo, urea, Vorges Proskauer, fenilalanina, movilidad, citrato de Simmons, descarboxilación de la arginina, lisina y ornitina y oxidación del ácido glucónico, para confirmar la presencia de E. coli.

\section{Identificación de Factores de Virulencia de STEC}

Las cepas compatibles con E. coli fueron sometidas a dos protocolos de PCR para detectar los factores de virulencia propios de STEC; uno de PCR múltiple para detectar $s t x 1$ y stx 2 , y uno de PCR simple para detec$\operatorname{tar} e a e \mathrm{~A}$.

Para ello se usaron cebadores descritos por Wu et al. (2010), que se muestran en el Cuadro 1. Se utilizó la técnica de extracción del DNA por shock térmico, descrita por Reischl et al. (2002). El volumen final de cada ensayo fue de $25 \mu 1$, conteniendo $0.75 \mu 1$ de $\mathrm{MgCl}_{2}, 2.5 \mu 1$ de buffer de PCR 10X, $1.25 \mu \mathrm{l}$ de cada cebador evaluado, $0.5 \mu 1$ dNTP 200 $\mu \mathrm{m}, 0.1 \mu \mathrm{T}$ Taq ADN polimerasa y $2 \mu \mathrm{l}$ de la muestra de ADN, ajustando el volumen final con agua bidestilada estéril. Las condiciones de los PCR fueron las descritas por Wu et al. (2010).

Para visualización de los productos amplificados se usó agarosa $1.5 \%$, colocando 5 $\mu 1$ del producto del PCR más $3 \mu 1$ del buffer de carga en cada pozo y se usó un voltaje de electroforesis de $90 \mathrm{~V}$ por $30 \mathrm{~min}$. Los geles se tiñeron en bromuro de etidio $\left(10 \mu \mathrm{g} \cdot \mathrm{ml}^{-1}\right) \mathrm{y}$ se visualizaron en un transluminador (Uvitec, Alemania).

\section{Análisis de Resultados}

Se determinó la presencia de STEC si las cepas de E. coli poseían al menos una de las Stxs. Se evidenció la diferencia estadística entre el tipo de superficie evaluada y la frecuencia de E. coli y STEC mediante la prueba de Chi cuadrado, usando el paquete estadístico SPSS 23. 
Cuadro 1. Cebadores usados para la detección de E. coli shigatoxigénica (STEC) en muestras de puestos de venta de carne de pollo

\begin{tabular}{clc}
\hline Gen & Secuencia 5' $-3^{\prime}$ & $\begin{array}{c}\text { Amplicón } \\
(\mathrm{bp})\end{array}$ \\
\hline \multirow{2}{*}{ eaeA } & AAACAGGTGAAACTGTTGCC & 454 \\
& $\begin{array}{l}\text { CTCTGCAGATTAACCTCTGC } \\
\text { CAACACTGGATGATCTCAG }\end{array}$ & \\
& $\begin{array}{l}\text { CAACCCCTCAACTGCTAATA } \\
s t x 2\end{array}$ & 349 \\
& ATCAGTCGTCACTCACTGGT \\
& CTGCTGTCACAGTGACAAA & 110 \\
\hline
\end{tabular}

Cuadro 2. Frecuencia de presentación de los genes $s t x 1$, stx 2 y eaeA en 63 cepas aisladas de E. coli de superficies de puestos de venta de carne de pollo (manos del trabajador, tabla de picar y mesa de expendio) en un mercado de abastos de San Juan de Miraflores, Lima

\begin{tabular}{lcccc}
\hline Cepas & $\begin{array}{c}\text { Manos de } \\
\text { trabajador }\end{array}$ & $\begin{array}{c}\text { Tabla de } \\
\text { picar }\end{array}$ & $\begin{array}{c}\text { Mesa de } \\
\text { expendio }\end{array}$ & $\begin{array}{c}\text { Total } \\
(\%)\end{array}$ \\
\hline$s t x 1$ & 5 & 3 & 4 & 19.0 \\
$s t x 2$ & 5 & 1 & 3 & 14.3 \\
$s t x 1+s t x 2$ & 1 & 2 & 5 & 12.7 \\
$e a e A$ & 1 & 4 & 0 & 7.9 \\
$s t x 1+s t x 2+e a e A$ & 1 & 2 & 1 & 6.3 \\
$s t x 2+e a e A$ & 1 & 2 & 0 & 4.8 \\
$s t x 1+e a e A$ & 2 & 0 & 0 & 3.2 \\
\hline Total & 16 & 14 & 13 & 68.3 \\
\hline
\end{tabular}

\section{Resultados}

El $42 \%(63 / 150)$ y $25.3 \%(38 / 150)$ de las muestras fueron positivas a $E$. coli y STEC, respectivamente. El 84\% (42/50) y $66 \%(33 / 50)$ de los puestos de venta poseían al menos una de las superficies contaminadas con E. coli y STEC, respectivamente; sin encontrarse diferencia estadística entre la presentación de E. coli o STEC y el tipo de superficie muestreada (Figura 1).
Se identificaron 63 cepas de E. coli, donde 21 fueron aisladas de manos de los trabajadores, 20 de tablas de picar y $22 \mathrm{de}$ mesas de expendio. El 60.3\% (38/63) de las cepas presentaron al menos un gen que codifica alguna de las dos Stxs. El 68.3\% (43/63) de las cepas de $E$. coli presentaron al menos un gen de virulencia evaluado (cepas patógenas) y el 6.3\% (4/63) de las cepas poseían los tres factores de virulencia. La frecuencia de cepas con factores de virulencia de interés en la salud pública se muestra en el Cuadro 2. 


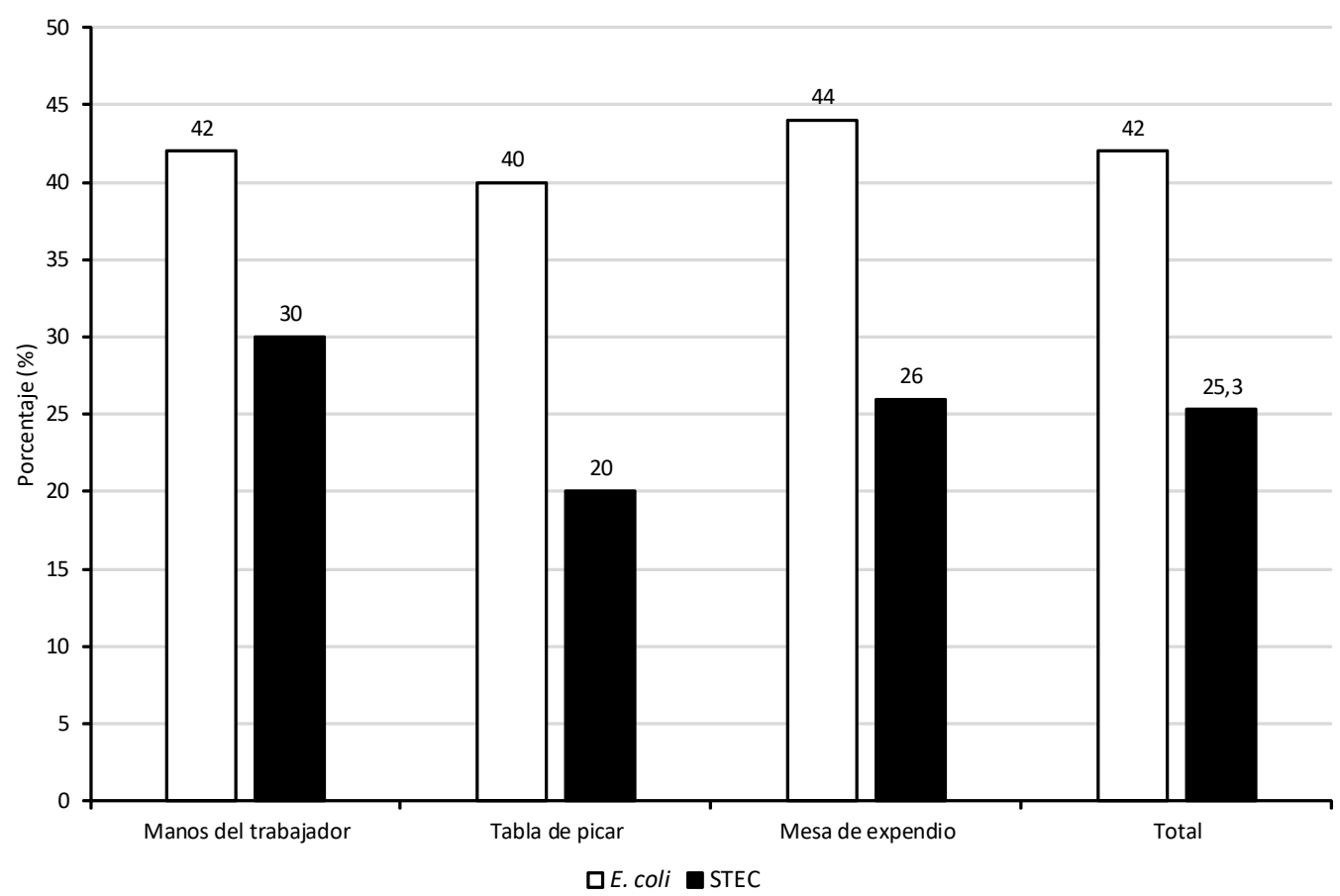

Figura 1. Frecuencia de E. coli y STEC en superficies de puestos de venta de carne de pollo (manos del trabajador, tabla de picar y mesa de expendio) en un mercado de abastos en San Juan de Miraflores, Lima (50 muestras por superficie)

Se observaron diversas deficiencias de buenas prácticas de manufactura durante el expendio en el puesto de venta. La mayoría de los vendedores no contaban con guantes, cobertores de cabello ni tapabocas. Tampoco disponían de un lavatorio apropiado donde lavarse las manos ni otro donde lavar y desinfectar los cuchillos y tablas con las debidas frecuencias.

En todos los puestos se observó canales de pollo sin eviscerar, la carne se vendía sin conservación en refrigeración y los manipuladores de la carne también realizaban el cobro. Además, los puestos de venta limitaban con otros puestos de venta de alimentos, como verduras, carne de pescado, especias y abarrotes.

\section{Discusión}

La presencia de STEC en al menos una de las superficies contaminadas del 66\% de los puestos de venta confirma al puesto de expendio de carne de pollo como fuente de contaminación con este agente.

Stxs son considerados factores de virulencia críticos en las enfermedades producidas por STEC. Stxs se unen a las células que poseen receptores afines bloqueando la síntesis proteica por su daño irreversible al ARN ribosomal (Endo et al., 1988). En el presente trabajo se determinó que $12.7 \%$ (8/63) de las cepas presentaron el gen $s t x 1$ y $s t x 2$, el $19.0 \%$ $(12 / 63)$ presentaron el gen $s t x 1$ y el $14.3 \%$ $(9 / 63)$ el gen $s t x 2$.

Diversos reportes señalan que el stx2 es el gen predominante de los STEC presente en varios niveles de la cadena de comercialización de la carne (Blanco et al., 2004). Stx2 es más tóxico para las células endoteliales microvasculares renales de los humanos que la Stx 1 (Louise y Obrig, 1995) y con mayor riesgo de desarrollar SUH (Paton y Paton, 2002; Ethelberg et al., 2004). 
Por otro lado, se ha descrito que la presencia en asociación de los genes eaeA y st $x 2$ resulta en una mayor severidad de la enfermedad (Ethelberg et al., 2004; Persson et al., 2007). El presente trabajo identificó el $4.8 \%(3 / 63)$ de cepas con esta combinación y $6.3 \%(4 / 63)$ que poseían los tres factores de virulencia evaluados. Además, el 3.2\% $(2 / 63)$ de las cepas presentaron eae A y $s t x 1$, lo cual también representa un problema de salud pública (Ethelberg et al., 2004; Gyles, 2007).

E1 7.9\% (5/63) de cepas de E. coli eran portadoras del gen eaeA y negativos a Stxs, cepas también patógenas para el hombre pues pueden ser del patotipo E. coli enteropatogénica, lo cual podría confirmarse identificando otros factores de virulencia no evaluados en este análisis (Nataro y Kaper, 1998), o incluso podría tratarse de cepas de STEC, que durante el cultivo y subcultivo perdieron las Stxs (Tomas et al., 2012).

La presencia de estos patógenos en el puesto de venta podría explicarse en gran medida por las prácticas de los manipuladores observadas durante el muestreo. En los mercados peruanos es común observar la venta de carne sin conservación en refrigeración y, más grave aún, la presencia de canales de pollo sin eviscerar, costumbre que el consumidor asocia a un estado de frescura de la carne. Es probable que esta mala práctica favorezca una contaminación desde la canal hacia el puesto de venta, con el riesgo inminente de una magnificación posterior de esta contaminación, como se ha observado en otros estudios (Samadpour et al., 1994; Chinen et al., 2009; Etcheverria et al., 2010; Alonso et al., 2012).

Algunos puestos poseían tablas de picar de madera, las cuales son inadecuadas por favorecer la formación de biopelículas y con ello la perpetuación del agente en el puesto. Algunas cepas de E. coli pueden desarrollar biopelículas como resultado de una mayor producción de exopolisacáridos (Ryu et al., 2004), la que las hacen resistentes incluso a soluciones de hipoclorito (Ryu y Beuchat, 2005; Wilks et al., 2005).

También debería considerarse la transmisión de E. coli y STEC hacia las carnes a través de moscas y desde fuentes externas como aire, suelo y agua (Keen et al., 2006). Asimismo, la recepción del dinero realizado por los manipuladores podría ser otra fuente de contaminación a tenerse en consideración (Ukwuru y Gabriel, 2012). Tampoco debería descartarse que los mismos vendedores pudieran fungir de reservorios de E. coli y STEC, y contaminar el puesto a través de sus manos y malas prácticas de higiene.

Es probable que, aunque el pollo usualmente no es un reservorio importante de STEC, el manejo en expendio y el puesto de venta favorezcan la contaminación de la carne con este agente, por lo que debería hacerse énfasis en la preparación cuidadosa de esta carne a nivel doméstico. Con estos resultados, debería fortalecerse el control de las buenas prácticas en el expendio, responsabilidad directa de los gobiernos locales y distritales, pues está demostrándose que la actual actividad es un riesgo de salud pública.

\section{Conclusiones}

Los puestos de venta de carne de pollo en el Mercado Cooperativo de Ciudad de Dios, del distrito de San Juan de Miraflores, Lima, es fuente potencial de contaminación con Escherichia coli y STEC.

\section{Literatura Citada}

1. Alonso MZ, Lucchesi PMA, Rodríguez EM, Parma AE, Padola NL. 2012. Enteropathogenic (EPEC) and Shigatoxigenic Escherichia coli (STEC) in broiler chickens and derived products at different retail stores. Food Control 23: 351-355. doi: 10.1016/j.foodcont. 2011.07.030 
2. Blanco JE, Blanco M, Alonso MP, Mora A, Dahbi G, Coira MA, Blanco $J .2004$. Serotypes, virulence genes, and intimin types of Shiga toxin (verotoxin)producing Escherichia coli isolates from human patients: prevalence in Lugo, Spain, from 1992 through 1999. J Clin Microbiol 42: 311-319.

3. Chinen I, Epszteyn S, Melamed CL, Aguerre L, Martínez-Espinosa E, Motter MM, et al. 2009. Shiga toxinproducing Escherichia coli $\mathrm{O} 157$ in beef and chicken burgers, and chicken carcasses in Buenos Aires, Argentina. Int J Food Microbiol 132: 167-171. doi: 10.1016/j.jifoodmicro.2009.03.021

4. Endo Y, Tsurugi K, Yutsudo T, Takeda Y, Ogasawara T, Igarashi K. 1988. Site of action of a Vero toxin (VT2) from Escherichia coli $\mathrm{O} 157: \mathrm{H} 7$ and of Shiga toxin on eukaryotic ribosomes. RNA Nglycosidase activity of the toxins. Eur J Biochem 171: 45-50. doi: 10.1111/j.14321033.1988.tb13756.x

5. Etcheverría AI, Padola NL, Sanz ME, Polifroni R, Krüger A, Passucci J, Rodriguez EM, et al. 2010. Occurrence of Shiga toxin-producing $E$. coli (STEC) on carcasses and retail beef cuts in the marketing chain of beef in Argentina. Meat Sci 86: 418-421. doi: 10.1016/j.meatsci.2010.05.027

6. Ethelberg S, Olsen KE, Scheutz F, Jensen C, Schiellerup P, Enberg J, Petersen AM, et al. 2004. Virulence factors for hemolytic uremic syndrome, Denmark. Emerg Infect Dis 10: 842-847. doi: 10.3201/eid1005.030576

7. Gyles CL. 2007. Shiga toxin-producing Escherichia coli: an overview. J Anim Sci 85: 45-62. doi: 10.2527/jas.2006-508

8. Keen JE, Wittum T, Dunn JR, Bono J, Durso LM. 2006. Shiga-toxigenic Escherichia coli $\mathrm{O} 157$ in agricultural fair livestock, United States. Emerg Infect Dis 12: 780-786.

9. Llanos A, Lee J, López, F, Contreras C, Barletta F, Chea-Woo E, Ugarte C, Cleary TG, Ochoa TJ. 2012. Shiga toxin-producing Escherichia coli in Peruvian children with bloody diarrhea. Pediatr Infect Dis J 31: 314-316. doi: 10.1097/INF.0b013e318244000c

10. Louise CB, Obrig TG. 1992. Shiga toxin-associated hemolytic uremic syndrome: combined cytotoxic effects of shiga toxin and lipopolysaccharide (endotoxin) on human vascular endothelial cells in vitro. Infect Immun 60: 1536-1543.

11. Lucas J, Vilca M, Ramos D. 2013. Presencia de Campylobacter spp en canales y ciegos de pollos de engorde en Lima, Perú. Rev Inv Vet Perú 24: 346352. doi: 10.15381/rivep.v24i3.2583

12. Nataro JP, Kaper JB. 1998. Diarrheagenic Escherichia coli. Clin Microbiol Rev 11: 142-201.

13. [MINSA] Ministerio de Salud del Perú. 2005. Manual de procedimientos bacteriológicos en infecciones intrahospitalarias. Lima: CEPREDIM. Serie de normas técnicas N. ${ }^{\circ} 28.106 \mathrm{p}$.

14. Paton AW, Paton JC. 2002. Direct detection and characterization of shiga toxigenic Escherichia coli by multiplex PCR for stx1, stx2, eae, ehxA and saa. J Clin Microbiol 40: 271-274. doi: 10.1128/ JCM.40.1.271-274.2002

15. Persson S, Olsen KE, Ethelberg S, Scheutz F. 2007. Subtyping methods for Escherichia coli Shiga toxin (verocytotocin) 2 variants and correlation to clinical manifestations. J Clin Microbiol 45: 2020-2024. doi : 10.1128/ JCM.02591-06

16. Piérard D, Van Damme L, Moriau L, Stevens D, Lauwers S. 1997. Virulence factors of verocytotoxin-producing Escherichia coli isolated from raw meats. Appl Environ Microbiol 63: 45854587.

17. Read SC, Gyles CL, Clarke RC, Lior H, McEwen S. 1990. Prevalence of verocytotoxigenic Escherichia coli in ground beef, pork, and chicken in southwestern Ontario. Epidemiol Infect 105: 11-20. doi: 10.1017/S0950268800047592 
18. Reischl U, Youssef MT, Kilwinski J, Lehn N, Zhang WI, Karch $H$, Strockbine N. 2002. Real-time fluorescence PCR assays for detection and characterization of Shiga toxin, intimin, and enterohemolysin genes from Shiga toxin-producing Escherichia coli. J Clin Microbiol 40: 2555-2565. doi: 10.1128/JCM.40.7.2555-2565.2002

19. Ryu JH, Beuchat LR. 2005. Biofilm formation by Escherichia coli $\mathrm{O} 157: \mathrm{H} 7$ on stainless steel: effect of exopolysaccharide and curli production on its resistance to chlorine. Appl Environ Microbiol 71: 247-254. doi: 10.1128/ AEM.71.1.247-254.2005

20. Ryu JH, Kim H, Beuchat LR. 2004. Attachment and biofilm formation by Escherichia coli O157:H7 on stainless steel as influenced by exopolysaccharide production, nutrient availability, and temperature. J Food Prot 67: 2123-2131.

21. Samadpour M, Ongerth JE, Liston J, Tran N, Nguyen D, Whittam TS, Wilson RA, Tarr PI. 1994. Occurrence of Shiga-like toxin-producing Escherichia coli in retail fresh seafood, beef, lamb, pork, and poultry from grocery stores in Seattle, Washington. Appl Environ Microbiol 60: 1038-1040.

22. Thomas KM, McCann MS, Collery MM, Logan A, Whyte P, McDowell $D A$, Duffy G. 2012. Tracking verocytotoxigenic Escherichia coli O157, O26, O111, O103 and O145 in Irish cattle. Int J Food Microbiol 153: 288-296. doi: 10.1016/j.ijfoodmicro.2011.11.012
23. Thorpe CM. 2004. Shiga toxinproducing Escherichia coli infection. Clin Infect Dis 38: 1298-1303. doi: 10. $1086 / 383473$

24. Ukwuru MU, Gabriel A. 2012. Cross contamination between food and money due to simultaneous handling. J Appll Sci Environ 3: 42-48.

25. Wilks SA, Michels H, Keevil CW. 2005. The survival of Escherichia coli $\mathrm{O} 157$ on a range of metal surfaces. Int $\mathrm{J}$ Food Microbiol 105: 445-454.

26. Wu Y, Hinenoya A, Taguchi T, Nagita A, Shima K, Tsukamoto T, Sugimoto $N$, et al. 2010. Distribution of virulence genes related to adhesins and toxins in shiga toxin-producing Escherichia coli strains isolated from healthy cattle and diarrheal patients in Japan. J Vet Med Sci 72: 589-597. doi: 10.1292/jvms.090557

27. Xia X, Meng J, McDermott PF, Ayers S, Blickenstaff K, Tran TT, Abbott J, et al. 2010. Presence and characterization of shiga toxin-producing Escherichia coli and other potentially diarrheagenic E. coli strains in retail meats. Appl Environ Microbiol 76: 17091717. doi: 10.1128/AEM.01968-09

28. Zambrano H, Lucas JR, Vilca M, Ramos D. 2013. Determinación de Salmonella spp en centros de beneficio clandestino de pollos de engorde en Lima, Perú. Rev Inv Vet Perú 24: $337-$ 345. doi: 10.15381/rivep.v24i3.2582 